\title{
A IMPORTÂNCIA DA CALIBRAGEM PARA AVALIAÇÃO RADIOGRÁFICA DE ANOMALIAS DENTÁRIAS DE PACIENTES COM CÂNCER
}

\author{
Ana Carolina Cunha Rodrigues* \\ Reyna Aguilar Quispe \\ Ana Lúcia Alvares Capelozza ${ }^{\star \star \star x}$ \\ Cássia Maria Fisher Rubira*t+o \\ Ana Maria Greff Buaes ${ }^{* * \alpha *}$ \\ Paulo Sérgio da Silva Santos ${ }^{\star *+\alpha+k}$
}

\begin{abstract}
RESUM0: Entende-se por anomalia uma variação ou desvio de uma característica ou estrutura anatômica, relativamente à normalidade. Pelo tratamento antineoplásico, sobreviventes de câncer infantil estão em risco de adquirir anomalias dentárias como efeito tardio. Esses efeitos podem ser observados pela radiografia panorâmica. Para resultados confiáveis e reprodutíveis é necessário que os examinadores passem por um treinamento prévio. Este pode ser medido por meio de testes de concordância como 0 teste de Kappa. 0 objetivo do presente trabalho foi apresentar a importância da calibragem dos examinadores para avaliação radiográfica de anomalias dentárias de pacientes com câncer, a fim de obter informações com alta confiabilidade. Foram selecionadas aleatoriamente 40 radiografias panorâmicas digitais, sendo 20 do grupo controle e 20 do grupo experimental. 0s examinadores foram considerados calibrados quando os valores do teste de Kappa estavam entre 0,61-1,0. A calibração intraexaminador teve como mínimo um valor de concordância substancial $(0,61-0,80)$ e um máximo de concordância perfeita $(0,81-1,0)$ para os dois examinadores. Em relação à calibração interexaminador observou-se como resultado mínimo de Kappa uma concordância moderada $(0,41-0,60)$ na primeira etapa, sendo que na segunda etapa chegou-se a uma concordância substancial $(0,61-0,80)$ demostrando que as examinadoras se encontravam calibradas. Para a avaliação de anomalias dentárias em radiografias panorâmicas é necessária uma calibração cautelosa e minuciosa que favoreça a emissão de informações confiáveis e reproduzíveis para evidência científica.
\end{abstract}

PALAVRAS-CHAVE: Reprodutibilidade dos testes; Diagnóstico; Pesquisa; Anormalidades dentárias; Radiografia panorâmica.

\section{THE IMPORTANCE OF CALIBRATION FOR RADIOGRAPHIC ASSESSMENT OF DENTAL ANOMALIES IN PATIENTS WITH CANCER}

\footnotetext{
ABSTRACT: Anomaly is a variation or deviation of an anatomic characteristic or structure from normality. Due to anti-cancer treatments, survival children with cancer are prone to have dental anomalies as a delayed effect revealed by full-mouth radiography. However, examiners should have previous training, assessed by concordance tests (Kappa test) for reliable and reproducible results. Calibration is important for examiners to evaluate dental anomalies in patients with cancer so that highly reliable data may be obtained. Forty full-mouth radiographies, twenty of control group and 20 of the experimental group, were randomly selected. Examiners proved to be calibrated when Kappa test rates were between 0.61and 1.0. Intra-examiner calibration featured between least

Discente da graduação da Faculdade de Odontologia de Bauru da Universidade de São Paulo, Departamento de Cirurgia, Estomatologia, Patologia e Radiologia da Faculdade de Odontologia de Bauru, Brasil.

** Mestranda de estomatologia na Faculdade de Odontologia de Bauru da Universidade de São Paulo, Departamento de Cirurgia, Estomatologia, Patologia e Radiologia da Faculdade de Odontologia de Bauru, Brasil.

${ }^{* * *}$ Docente. Dra. da Faculdade de Odontologia de Bauru da Universidade de São Paulo, Departamento de Cirurgia, Estomatologia, Patologia e Radiologia da Faculdade de Odontologia de Bauru, Brasil

${ }^{* * * *}$ Docente. Dra.da Faculdade de Odontologia de Bauru da Universidade de São Paulo, Departamento de Cirurgia, Estomatologia, Patologia e Radiologia da Faculdade de Odontologia de Bauru, Brasil

${ }^{* * * * *}$ Coodenadora de Odontologia do Instituto de Câncer Infantil, Departamento de odontologia do Instituto de Câncer Infantil, Brasil

****** Docente. Dr. da Faculdade de Odontologia de Bauru da Universidade de São Paulo, Departamento de Cirurgia, Estomatologia, Patologia e Radiologia da Faculdade de Odontologia de Bauru, Brasil. E-mail: paulosss@fob.usp.br
} 
(0.61-0.80) and maximum concordance $(0.81-1.0)$ for the two examiners. In the case of inter-examiner calibration, minimum Kappa featured moderate concordance $(0.41-0.60)$ during the first stage; whilst a substantial concordance was reached $(0.61-$ 0.80 ) in the second stage, proving standardization of examiners. A careful and detailed calibration is required to evaluate dental anomalies in full-mouth radiographies, enhancing reliable and reproducible information for scientific evidence.

KEYWORDS: Reproducibility of tests; Diagnosis; Research; Dental anomalies; Orthopantomography.

\section{INTRODUÇÃO}

Os métodos tradicionais de tratamento oncológico são a cirurgia, a radioterapia e a quimioterapia. 0 tratamento depende da localização, grau de malignidade, estadiamento clínico do tumor e condição de saúde do indivíduo. (SENA et al., 2001; DE CASTRO et al., 2002). A literatura relata que 0 tratamento antineoplásico pode causar efeitos agudos e tardios na cavidade bucal, sendo que na infância 0 desenvolvimento dos dentes pode ser afetado causando dano celular tanto nos odontoblastos quanto nos amelobastos (MOLLER; PERRIER, 1998; YAMAMOTO et al., 2012).

Para que seja possível visualizar as anomalias de desenvolvimento dentário de forma abrangente, a radiografia panorâmica é 0 exame de imagem convencional indicado. A radiografia panorâmica é um exame complementar utilizado na avaliação epidemiológica de diversas condições mórbidas que acometem 0 terço médio-inferior da face (GIRONDI et al., 2006).

Os achados radiográficos, como parte das alterações dentárias decorrentes do tratamento antineoplásico, compreendem as anomalias de desenvolvimento como hipodontia, microdontia, fusão, taurodontismo e anomalias na raiz dentária entre outros (GIRONDI et al., 2006, PROC; SZCZEPA; SKIBA, 2015).

É condição fundamental para a confiabilidade dos dados manter a boa reprodutibilidade das observações pela subjetividade inerente da radiografia panorâmica. Para tal efeito, utiliza-se o treinamento de calibração dos examinadores (MINISTÉRIO DA SAÚDE, 2009).

Analisando o contexto acerca das anomalias dentárias e a variação na interpretação de radiografias panorâmicas, 0 objetivo do presente trabalho foi apresentar a importância da calibragem para avaliação radiográfica de anomalias dentárias de pacientes com câncer, a fim de obter informações com alta confiabilidade.

\section{METODOLOGIA}

\subsection{SELEÇÃO DO MATERIAL}

Foram selecionadas aleatoriamente 40 radiografias panorâmicas digitais com boa qualidade de imagem, sendo 20 de indivíduos sem histórico de câncer grupo controle (GC) e 20 de indivíduos que passaram por tratamento antineoplásico durante a infância, grupo experimental (GE). A fim de evitar viés, os examinadores não tiveram acesso a informações adicionais como idade, sexo e/ou doença de base. As radiografias foram realizadas em diferentes aparelhos de raio-x panorâmico, sendo assim foram adicionados quadros escuros nos cantos inferiores de todas as imagens para cobrir a marca do aparelho utilizado (Figura 1).

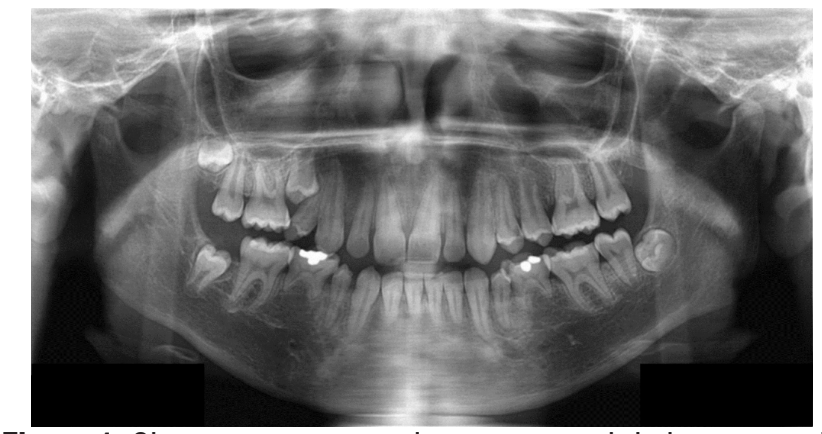

Figura 1. Observam-se os quadros escuros cobrindo a marca do aparelho panorâmico 


\subsection{ESCOLHA DO LOCAL}

A avaliação das radiografias panorâmicas foram realizadas no laboratório de radiologia da Faculdade de Odontologia de Bauru, que conta com monitores utilizados para avaliação radiográfica (SAMSUNG, modelo SyncMaster 740B), assim como iluminação apropriada para este tipo de estudos radiográficos.

\subsection{ANOMALIAS DENTÁRIAS AVALIADAS}

Para a avaliação destas anomalias foi utilizada a classificação de Alvares e Tavano (2009) sendo:

- alterações

hiperplasiantes:

dentes supranumerários, raízes supranumerárias, fusão, concrescência, geminação dentária, macrodontia e taurodontismo;

- alterações hipoplasiantes: anodontia, hipodontia e microdontia;

- alterações heterotópicas: giroversão, transposição dentária, transmigração dentária, dilaceração dentária, dente invaginado e dentes não irrompidos.

\subsection{TABULAÇÃO DOS DADOS}

Foi confeccionada uma planilha de Excel que abrangesse todos os itens que seriam observados em cada radiografia, sendo que a cada etapa da análise era utilizada uma planilha em branco.

\subsection{AVALIAÇÃO RADIOGRÁFICA}

A análise foi dividida em duas etapas, que ocorreram com 15 dias de diferença, utilizando uma planilha em branco em cada caso. Todas as análises foram realizadas por duas examinadoras, concomitantemente, em computadores separados, sem comunicação e realizando a contagem de tempo.

\subsection{CALIBRAÇÃO}

A calibração foi realizada por duas professoras experientes em radiologia, em momentos distintos. A primeira calibração ocorreu de forma eletrônica, com um questionário que continha exemplos de todas as anomalias dentárias avaliadas neste estudo. As examinadoras preencheram 0 questionário que foi enviado para a professora responsável, sendo que com resultados satisfatórios foram liberadas para iniciar o teste de Kappa. Para minimizar os erros de análise, as examinadoras foram submetidas à nova calibração, com uma segunda professora de radiologia, que repassou pessoalmente cada um dos itens de anomalias dentárias, mostrando exemplos e retirando as dúvidas que existiam. Finalizada a calibração, as examinadoras foram submetidas à análise de concordância intraexaminador (cálculo realizado sobre a concordância entre o mesmo examinador) e interexaminador (cálculo realizado sobre a concordância entre os examinadores) por meio do teste de Kappa tomando em conta os seguintes valores: $<0$ (não existe concordância); 0-0,20 (concordância mínima); 0,21-0,40 (concordância razoável); 0,41 - 0,60 (concordância moderada); 0,61-0,80 (concordância substancial); 0,81 -1,0 (concordância perfeita). 0s examinadores foram considerados calibrados quando os valores do teste de Kappa estavam entre 0,611,0. (concordância substancial-concordância perfeita) (LÓPEZ; PITA, 1999).

\subsection{ANÁLISE ESTATÍSTICA}

0 valor do teste de Kappa foi convertido a percentual para avaliar o nível de concordância entre 0 examinador 1 e 2 , respeito às variáveis qualitativas nominais como anomalias dentárias hipoplasiantes, hiperplasiantes e heterotópicas que foram descritas por meio da porcentagem. 


\section{RESULTADOS E DISCUSSÃO}

Foram avaliadas 40 radiografias panorâmicas, sendo 20 do GEe 20 do GC. 0 teste de Kappa intraexaminador teve como mínimo o valor de 0,85 (concordância substancial) e um máximo de 1 (concordância perfeita) no examinador 1. Entretanto, 0 valor mínimo foi de 0,68 (concordância substancial) e 0 máximo de 1 (concordância perfeita) no examinador 2 (Tabela 1).

Tabela 1. Teste Kappa intra-examinador das anomalias dentárias

\begin{tabular}{|c|c|c|c|c|}
\hline \multirow[b]{2}{*}{ HIPERPLASIANTES } & \multicolumn{2}{|c|}{ EXAMINADOR 1} & \multicolumn{2}{|c|}{ EXAMINADOR 2} \\
\hline & & & & \\
\hline Variável & \%Concordância & Kappa & \%Concordância & Kappa \\
\hline Supranumerário & 100 & 1 & 100 & 1 \\
\hline Raiz supranumerária & 100 & 1 & 97,5 & 0,98 \\
\hline Fusão & 97,44 & 0,97 & 100 & 1 \\
\hline Concrescência & 100 & 1 & 100 & 1 \\
\hline Geminação & 100 & 1 & 100 & 1 \\
\hline Macrodontia & 100 & 1 & 97,5 & 0,98 \\
\hline Taurodontismo & 97,5 & 0,98 & 97,5 & 0,98 \\
\hline \multicolumn{5}{|l|}{ HIPOPLASIANTES } \\
\hline Anodontia & 100 & 1 & 100 & 1 \\
\hline Hipodontia & 95 & 0,95 & 90 & 0,90 \\
\hline Microdontia & 100 & 1 & 95 & 0,95 \\
\hline \multicolumn{5}{|l|}{ HETEROTOPICAS } \\
\hline Giroversão & 85 & 0,85 & 67,5 & 0,68 \\
\hline Transposição & 100 & 1 & 100 & 1 \\
\hline Transmigração & 100 & 1 & 97,5 & 0,98 \\
\hline Dilaceração & 92,5 & 0,93 & 82,5 & 0,83 \\
\hline Invaginado & 100 & 1 & 97,5 & 0,98 \\
\hline Não Irrompido & 100 & 1 & 92,5 & 0,93 \\
\hline
\end{tabular}

Examinador 1: Aluna de Pós-Graduação e Examinador 2: Aluna de Graduação

A concordância interavaliador teve um valor de Kappa mínimo de 0,48 (concordância moderada) e máxima de 1 (concordância perfeita) na primeira etapa. Na segunda etapa após 15 dias de intervalo, 0 valor de Kappa mínimo foi 0,73 (concordância substancial) e máxima de 1 (concordância perfeita) (Tabela 2).

Tabela 2. Teste Kappa inter-examinador das anomalias dentárias

(Continua)

ETAPA 1

ETAPA 2

\begin{tabular}{lcccc}
\hline HIPERPLASIANTES & \multicolumn{3}{c}{} \\
\hline Variável & \%Concordância & Kappa & \%Concordância & Kappa \\
\hline Supranumerário & 100 & 1 & 90 & 0,9 \\
Raiz supranumerária & 88,5 & 0,88 & 95 & 0,95 \\
Fusão & 97,5 & 0,98 & 100 & 1 \\
Concrescência & 100 & 1 & 100 & 1 \\
Geminação & 100 & 1 & 100 & 1 \\
Macrodontia & 97,5 & 0,98 & 100 & 1 \\
Taurodontismo & 92,5 & 0,93 & 85 & 0,85 \\
\hline HIPOPLASIANTES & & & & \\
\hline Anodontia & 100 & 1 & 100 & 1 \\
Hipodontia & 85 & 0,85 & 90 & 0,9 \\
Microdontia & 92,5 & 0,93 & 85 & 0,85 \\
\hline HETEROTOPICAS & & & & \\
\hline Giroversão & 72,5 & 0,73 & 67,5 & 0,68 \\
\hline
\end{tabular}


(Conclusão)

\begin{tabular}{lcccc}
\hline Variável & \%Concordância & Kappa & \%Concordância & Kappa \\
\hline Transposição & 100 & 1 & 100 & 1 \\
Transmigração & 100 & 1 & 97,5 & 0,98 \\
Dilaceração & 47,54 & 0,48 & 72,5 & 0,73 \\
Invaginado & 70 & 0,7 & 80 & 0,8 \\
Não Irrompido & 90 & 0,9 & 87,5 & 0,88 \\
\hline
\end{tabular}

Etapa 1 e Etapa 2 foram realizadas com 15 dias de diferença.

A reprodutibilidade é descrita como 0 grau de concordância dos resultados quando a análise se repete, em condições idênticas (B00TH et al., 2002). Espera-se um erro mínimo aleatório ou sistemático, dentro das variações aceitas para uma variável particular, demonstrando que a medida de aferição utilizada é capaz de avaliar o que foi planejado (SOUZA et al., 2011). Neste estudo, a calibração intraexaminador tanto para 0 examinador 1 quanto para 0 examinador 2 teve como mínimo um valor de concordância substancial e um máximo de concordância perfeita. É importante ressaltar que 0 treinamento das examinadoras foi realizado com dois professores experientes de radiologia, o que provavelmente permitiu chegar aos valores de Kappa satisfatórios. Em relação à calibração interexaminador mostrou-se concordância moderada como mínimo, sendo que na segunda etapa chegou-se como mínimo a uma concordância substancial demostrando que as examinadoras se encontravam calibradas.

A calibração tem como objetivo diminuir as discrepâncias entre um ou mais examinadores por meio da repetição de exames nas mesmas pessoas, pelos mesmos avaliadores em tempos diferentes. Dessa maneira objetiva-se assegurar que estão sendo utilizados os mesmos critérios para observar e registrar os dados. Com o padrão adotado e testado garante-se menor variação entre avaliadores (ORGANIZAÇÃO MUNDIAL DA SAÚDE, 1991, WORLD HEALTH ORGANIZATION, 1997, PERES; TRAEBERT; MARCENES, 2001).

As divergências de diagnóstico podem ocorrer entre os diferentes examinadores (interexaminadores) ou entre os diferentes exames de um mesmo grupo de pessoas, por um mesmo examinador ao longo do tempo (intraexaminador) (PERES; TRAEBERT; MARCENES, 2001). No caso do presente estudo, as maiores divergências foram relacionadas a anomalias dentárias como giroversão e a dilaceração tanto no quesito intraexaminador, quanto para interexaminador. Mesmo assim, a definição de critérios para observação das imagens após a calibragem foi 0 que permitiu resultados satisfatórios do Kappa para que fosse possível a continuação da pesquisa em questão.

Segundo Brito et al. (2012), das 71 radiografias analisadasemseuestudo,foramencontrados 246 dentes aparentemente com giroversão, sendo que $48,6 \%$ das giroversões identificadas radiograficamente eram "virtuais". Isto pode ocorrer quando a dinâmica de giro do eixo do equipamento panorâmico não coincide com a curvatura da maxila e mandíbula, a imagem dos dentes apresenta-se girovertida ou com apinhamento dental. As discrepâncias sobre giroversão no estudo estão relacionadas à adaptação dos examinadores com respeito às imagens analisadas, demonstrando que num primeiro momento muitos dentes foram classificados como girovertidos, sendo que na próxima análise houve maior refinamento quanto às variáveis que devem ser levadas em consideração ao analisar as radiografias panorâmicas.

A baixa qualidade de radiografias panorâmicas foi apontada como responsável pela redução na certeza de diagnósticos, desacreditando a radiografia como instrumento de diagnóstico pela Organização Mundial da Saúde (OMS). Para evitar interpretação errônea de patologias e estruturas anatômicas é essencial que 
se obtenha uma imagem de qualidade, seja para 0 planejamento do tratamento de patologias ou para pesquisas odontológicas (SCHIFF etal., 1986; CONSOLO et al., 1990; YACOVENCO, 2001; ANDRADE et al., 2003; CARVALHO et al., 2009). Para que as radiografias panorâmicas fossem selecionadas para 0 estudo era necessário que estas fossem de boa qualidade. Apesar disso, uma desvantagem desse tipo de radiografia é a sobreposição de imagens, que pode confundir 0 examinador. Portanto, o treinamento de avaliação de radiografias é indispensável para desenvolver 0 senso crítico na identificação de anomalias dentárias.

\section{CONSIDERAÇÕES FINAIS}

Para a avaliação de anomalias dentárias em radiografias panorâmicas é necessária uma calibração que favoreça a emissão de informações confiáveis e reprodutíveis para evidência científica. Diante dessa necessidade é importante que todos os aspectos metodológicos sejam levados em consideração para melhorar a qualidade dos estudos. Segundo os resultados notou-se como a utilização do teste de Kappa pode orientar os examinadores sobre a necessidade de treinamento antes de iniciar a coleta de dados, propriamente dita. Portanto, para análise das anomalias dentárias em radiografias panorâmicas é relevante que sejam utilizados testes de avaliação da calibragem dos examinadores, uma vez que 0 diagnóstico precoce e assertivo de uma determinada anomalia dentária pode orientar 0 cirurgião-dentista acerca do plano de tratamento desse paciente, garantindo-Ihe melhor qualidade de vida.

\section{REFERÊNCIAS}

ALVARES, L. C.; TAVANO, 0. Curso de radiologia em Odontologia. 5. ed. São Paulo: Santos, 2009. 274p.

ANDRADE, E. L. S.; CAVALCANTI, Y. S. L; LIMA, V. P.; FERREIRA, E. F.; FREIRE, A. R. S. Avaliação das radiografias periapicais: estudo realizado na clínica de graduação da Faculdade de Odontologia da UFMG. Arq Odontologia, Belo Horizonte, v. 39, n. 2, p. 75-162, abr./jun. 2003.

BOOTH, M. L.; OKELY, A. D.; CHEY, T. M.; BAUMAN, A. The reliability and validity of the Adolescent Physical Activity Recall Questionnaire. Med Sci Sports Exerc, Indianapolis, v. 34, n. 12, p. 1986-1995, dec. 2002.

BRITO, C. P.; COCLETE, G. A.; SALZEDAS, L. M. P.; CARVALHO, A. A. F.; PAULON, S. S.; COCLETE, G. E. G.; RAMOS, A. P. B. Giroversão dental: formação de imagem radiográfica virtual. Archives of Health Investigation, v. 1, n. 2012 s1, p. 30, 2012. Disponível em: <http:// hdl.handle.net/11449/133555>. Acesso em: 05 ago. 2017.

CARVALHO, P. L.; NEVES, A. C. C.; ZOLLNER, N. A.; ROSA, L. C. L.; ALMEIDA, E. T. D. C.; MEDEIROS, J. M. F. Erros técnicos nas radiografias intrabucais realizadas por alunos de graduação. RG0, v. 57, n. 2, p. 151-155, abr./jun. 2009.

CONSOLO, C. G.; MONTEBELO FILHO, A.; TAVANO, 0. Avaliação do desempenho de alunos de graduação na obtenção de radiografias pela técnica da bissetriz, durante os anos de 1986 e 1987. Rev. Odontol. USP, v. 4, n. 3, p. 247-251, jul./set. 1990.

DE CASTRO, R. M.; DEZOTTI, M.; AZEVEDO, L.; AQUILANTE, A.; XAVIER, C. R. Atenção odontológica aos pacientes oncológicos antes, durante e depois do tratamento antineoplásico. Rev Odontol UNICID, v. 14, n. 1, p. 63-74, 2002.

GIRONDI, J. R.; FENYO-PEREIRA, M.; CAMPOS, P. S. F.; PANELLA, J. Estudo da prevalência das anomalias dentárias de desenvolvimento em dada população, com 0 uso de radiografias panorâmicas. Revista de Odontologia da Universidade Cidade de São Paulo, v. 18, n. 1, p. 15-21, jan./abr. 2006. 
LÓPEZ, I. U. G.; PITA, S. F. Medidas de concordancia: el índice de kappa, Espanha, Cad aten primaria, v. 6, p. 169- 71, 1999.

MINISTÉRIO DA SAÚDE. SAS/S VS Depto de Atenção Básica Coordenação Nacional de Saúde Bucal. Manual de Calibração de examinadores. Brasília, 2009. 21p.

MOLLER, P.; PERRIER, M. Dento-maxillofacial sequelae in a child treated for a rhabdomyosarcoma in the head and neck. A case report. Oral surgery, oral medicine, oral pathology, oral radiology, and endodontics, v. 86, n. 3, p. 297-303, 1998.

ORGANIZAÇÃO MUNDIAL DA SAÚDE. Levantamento epidemiológico básico de saúde bucal. 3. ed. São Paulo: Santos; 1991.

PERES, M. A.; TRAEBERT, J.; MARCENES, W. Calibração de examinadores para estudos epidemiológicos de cárie dentária. Cadernos de Saúde Pública, Rio de Janeiro, v. 17, n. 1, p. 153-159, jan./fev. 2001.

PROC, P.; SZCZEPA, J.; SKIBA, A. Dental Anomalies as Late Adverse Effect among Young Children Treated for Cancer. Cancer Res Treat., v. 48, n. 2, p. 658-667, 2015.

SCHIFF, T.; D’AMBROSI0, J.; GLASS, B. J.; LANGLAIS, R. P.; MCDAVID, .W. D. Common positioning and technical errors in panoramic radiography. J Am Dent Assoc., v. 113, n. 3, p. 422-426, sep. 1986.

SENA, C.; SOUZA, F.; MORAIS, L.; PINTO, L.; MELO, N. Protocolo de conduta para tratamento de pacientes portadores de câncer bucal que realizarão radioterapia. FOA, v. 3, n. 1, p. 62-66, 2001.

SOUZA, J. A.; PASINATO, F.; BASSO, D.; CORREIA, E. C. R.; SILVA, A. T. Biofotogrametria confiabilidade das medidas do protocolo do software para avaliação postural (SAP0). Rev bras cineantropom desempenho hum, Florianópolis, v. 13, n. 4, p. 299-305, 2011.

WORLD HEALTH ORGANIZATION. Oral Health surveys: basic methods. 4. ed. Geneva: ORH EPID, 1997.

YACOVENCO, A. Análise dos problemas mais freqüentes da radiografia na prática odontológica. Revista da ABR0, v. 2, n. 1, p. 29-39, jan./jun. 2001.
YAMAMOTO, T.; KITA, M.; MORI, M.; AKAMATSU, Y.; ICHIOKA, H.; IEHARA T.; HOSOI, H.; KANAMURA, N. Antitumor chemotherapeutic agents induce developmental disorders of tooth germ. Journal of Oral and Maxillofacial Surgery, Medicine, and Pathology, v. 24, n. 4, p. 208-212, 2012.

Recebido em: 04 de setembro de 2017 Aceito em: 26 de outubro de 2017 